\title{
Immunization in Canada 2007
}

\author{
Joanne Embree MD FRCPC
}

$\mathrm{T}^{\mathrm{h}}$ he 7th Canadian Immunization Conference was held in Winnipeg, Manitoba, in December 2006. The theme of the conference was "Celebrating Immunization in Canada". It was clearly appropriate. Just four years previously, organizers of the 5th Canadian Immunization Conference held in Victoria, British Columbia, had devoted the meeting toward advocacy for the establishment of a national immunization strategy directed toward a doubtful civil service. How quickly and completely the immunization landscape in Canada changed! The proposed immunization strategy was accepted shortly thereafter. Also, its development was adequately funded. The fact that the federal government recently renewed its commitment to long-term funding support for the federal administrative component shows that a central coordinating function for immunization is seen as being important. Importantly, three years previously, a $\$ 300$ million trust fund was set up to allow the provinces and territories to introduce four new immunization programs. These included programs with varicella vaccines, adolescent and adult acellular pertussis vaccines, infant and child pneumococcal conjugate vaccines, and infant and adolescent conjugate meningococcal $\mathrm{C}$ vaccines. All provinces and territories have now successfully introduced these programs using the funds from the trust fund. Those provinces that introduced their programs two or more years previously are also beginning to see the benefits of the immunization programs including a reduced burden of illness and, specifically, documented reduced hospitalizations due to these infections. In addition, over this time period, the Public Health Agency of Canada was formed in response to the severe acute respiratory syndrome epidemic. It is beginning to change the manner in which public health activities are being conducted in Canada, as well as how Canada's public health community interacts with its counterparts in the rest of the world. Immunization is one of the priorities of the Public Health Agency of Canada.

The progress over the past four years has definitely been a well-known success story for immunization in Canada. Unfortunately, that may be a problem. Immunization programs always seem to suffer when they are successful. We usually refer to this problem when discussing the issue of parental reluctance to immunize their children because the prevalence of the diseases that the vaccines protect against is so low that parents are unfamiliar with the risks the diseases pose to their children. In this instance, however, the much publicized gains that have been made in the immunization programs run the risk of being 'checked off' by advocacy groups and governments alike the way accomplishments that have been completed are when, in fact, they have not been, and further improvements and initiatives are still needed.
Most importantly, the trust fund set up three years previously to assist in the introduction of new immunization programs sunsets this year. At the time of writing the present article, there was no indication that it will be renewed or that a new trust fund will be developed. This issue is important because there are two new immunization programs currently waiting to be introduced: human papillomavirus immunization of preadolescent and adolescent girls, and rotavirus immunization of infants. The vaccines that have recently been approved for use in these programs are the result of considerable scientific innovation and study by the pharmaceutical companies that developed them and, thus, the programs will be costly to introduce. Also, the human papillomavirus vaccine presents a new challenge for immunization due to the fact that, although the major thrust for its use is to prevent cervical and other genital cancers, the vaccine works by preventing infection with a sexually transmitted pathogen. To be beneficial, it must be provided before the individual's sexual debut. Thus, to be successful, the programs need to be prepared to deal with potential parental reluctance to deal with their preadolescent child's future sexuality. Thus, the cost of a public awareness campaign will be an additional necessary part of the introduction of these programs.

Although all provinces and territories have introduced newer vaccines with the help of the trust fund, it was still inadequate to allow all of the jurisdictions to include 'catch-up' programs along with the institution of funding for routine immunization according to the established schedules. Thus, there was an intrinsic inequity in the program and the nonimmunized children were reliant on the herd immunity effect to reduce the prevalence of the pathogen within their community. This can take some time, and so these children have remained at risk for some time after the immunization introduction, unless their parents were prepared to personally pay for their immunizations. For those susceptible children not immunized against varicella, this could have serious consequences in the future because adults are more likely to have more severe disease or complications. Therefore, hopefully, a new trust fund will provide funding for introduction of new vaccines, as well as for publicly funded comprehensive catch-up campaigns for those vaccines for which it would be appropriate.

The national immunization strategy has not, in fact, been fully developed. The immunization goal-setting aspect of the strategy has only developed generalized goals for a few vaccines. In reality, this activity should be the base on which the immunization strategy functions. We need to set specific, vaccine-preventable disease goals for each vaccine-preventable disease. These goals should specify whether the aim is to 
eliminate the pathogen in Canada (or worldwide if applicable), or eliminate or minimize the disease incidence in Canada. Without defining these parameters, how do we determine how best to use the vaccines in the optimal effective (including cost-effective) manner? In some instances, other interventions will be needed in addition to vaccines to accomplish disease-specific goals. By determining the goals first, these interventions can be included in the plans to accomplish the country's goals for disease elimination or control. We also need these goals to be clearly defined to determine the appropriate methods of monitoring the diseases and vaccine use. In doing so, we will probably find that our current programs of disease and vaccine monitoring will need to be supported and will likely need to be expanded and enhanced to do this appropriately. We need to use the immunization strategy to enhance existing monitoring systems to better determine who is not receiving the vaccines and develop a comprehensive programmatic research agenda to determine why this is so.

The National Advisory Committee on Immunization always tries to use evidence-based methods to assess whether a vaccine should be used and the best schedule for its use. This information is generally supplied by the pharmaceutical company that developed and tested the particular vaccine for which recommendations for use are being made. The studies performed are generally well-powered to show the effectiveness of the vaccines among the general healthy population in the countries in which the vaccines were tested. However, the National Advisory Committee on Immunization frequently has to extrapolate data from other sources to make recommendations for other populations who were not part of the vaccine study groups but for whom the vaccines could be beneficial (ie, immune-suppressed persons, persons with other chronic diseases and pregnant women). There is a need to support research related to the use of vaccines within these vulnerable populations in Canada. Also, because a number of the newer vaccines are designed to target specific strains of the pathogen, it is imperative that we determine whether the strains included in the vaccine are responsible for a significant percentage of the diseases in Canada, both before the institution of the vaccine program and after it has been put into place. We need to ensure that the immunization strategy includes appropriate funding for this activity. Canada has a talented basic science medical microbiology and immunology community. Scientists within this community are working hard to determine new vaccine methodologies. However, public funding to support the research activities of these scientists is extremely modest compared with that provided by other countries. Hopefully, through the immunization strategy, funding for vaccine research can be significantly increased.

The vaccine schedules across the country can still be described as a 'patchwork quilt'. The Canadian Immunization Committee will hopefully help to harmonize the vaccine schedules across the country. For children and parents who move from province to province, this can result in missed immunizations, and a national immunization registry could help ensure that this is minimized. Until that time, we are in a situation in which we can study the effectiveness of various immunization schedules in the prevention of vaccine-preventable diseases in Canadian children by comparing the successes and failures of each program, and this should be supported through the immunization strategy.

Thus, while we should celebrate the incredible progress in improving the immunization programs provided in Canada, we should not stop the efforts to improve it further. 


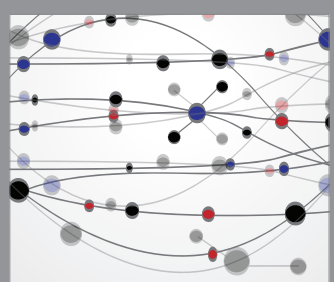

The Scientific World Journal
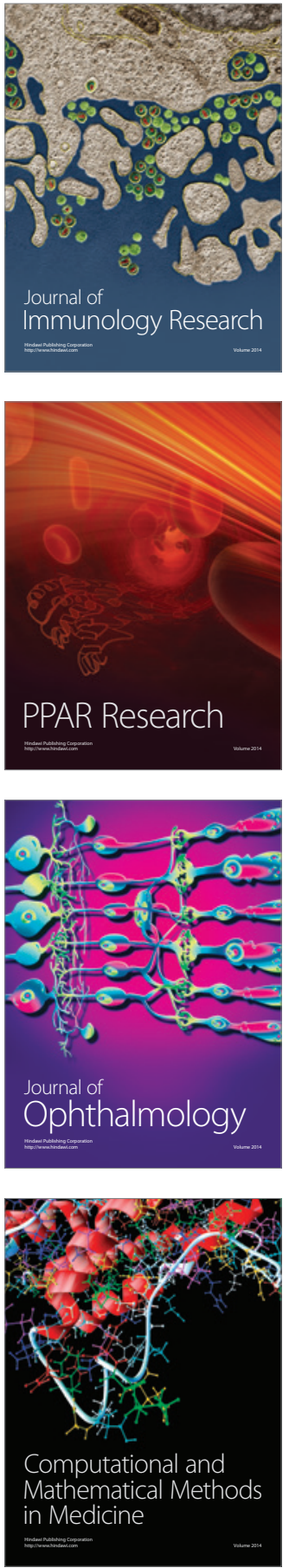

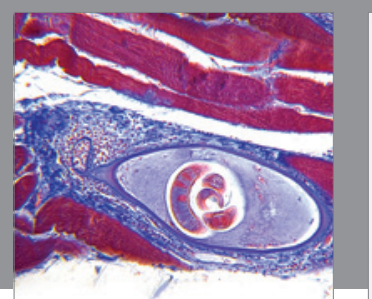

Gastroenterology Research and Practice

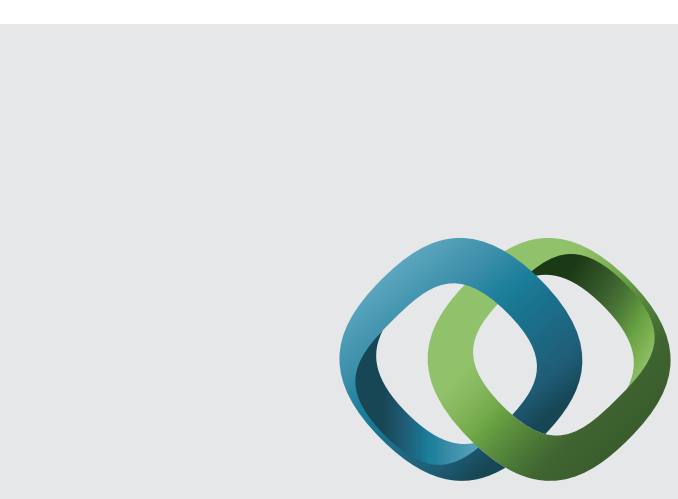

\section{Hindawi}

Submit your manuscripts at

http://www.hindawi.com
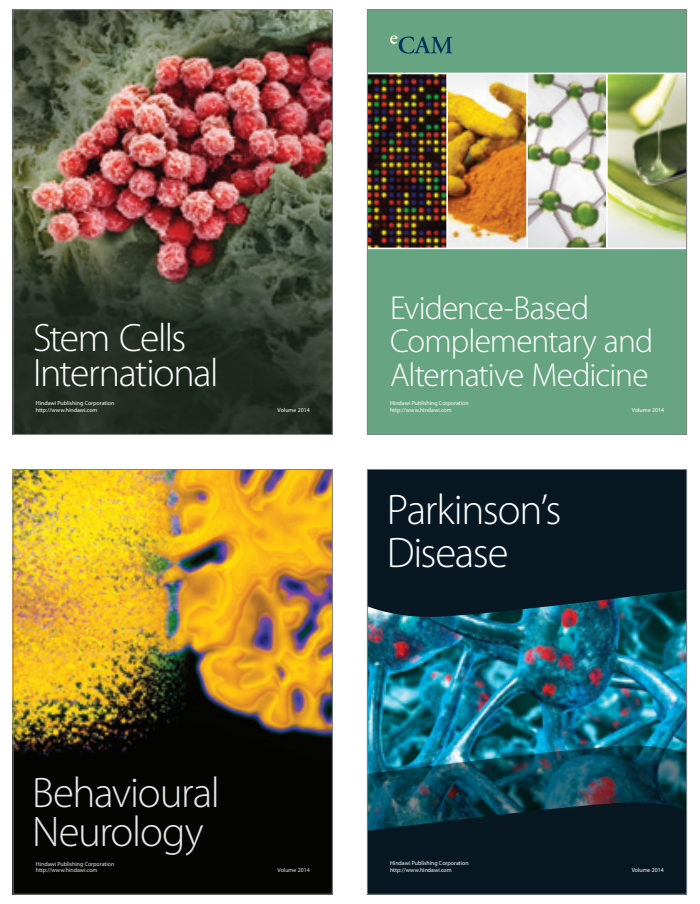
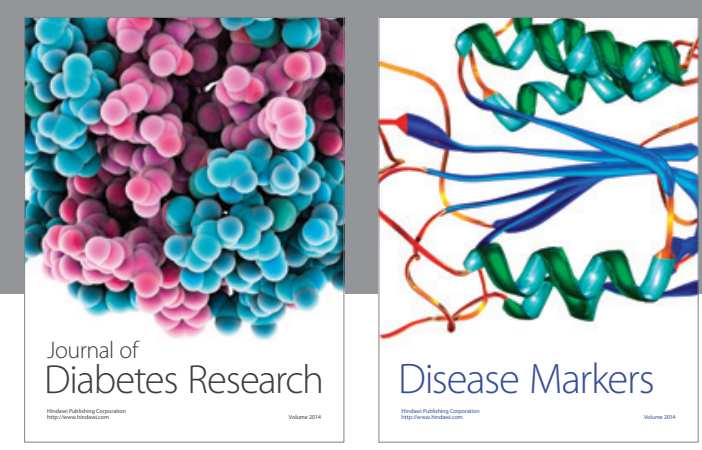

Disease Markers
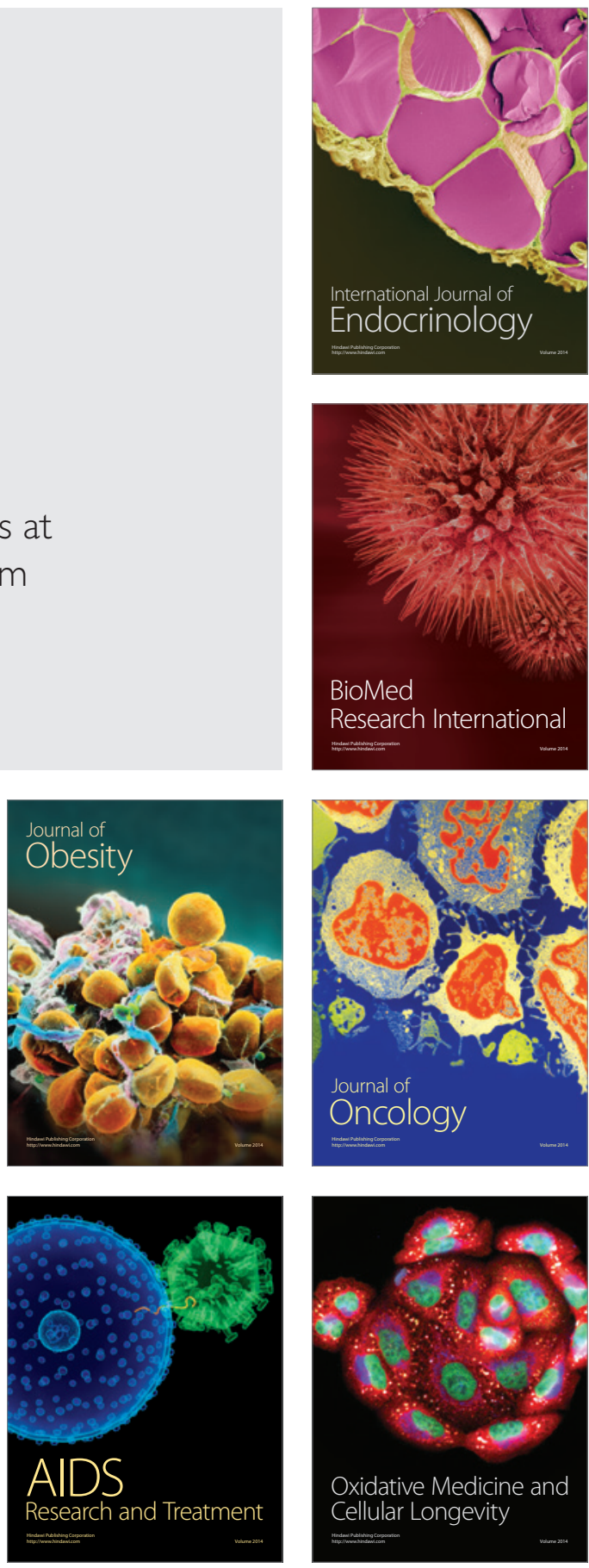